(Ior) V. G. Fesenkov, D. A. Rozhkovski et al., C.R. Acad. Sci. U.R.S.S. 88, no. 3, 1953.

(102) V. G. Fesenkov, D. A. Rozhkovski et al., Astr. J. U.S.S.R. 3r, no. 3, 1954.

(103) S. B. Pikelner, Publ. Crim. Obs. 12, 93, 1954.

(104) J. W. Chamberlain, Ap.J. 117, $3^{87}$ and 399, 1953.

(I05) R. Minkowski, Camb.

(I06) M. Johnson, Mon. Not. R. Astr. Soc. 113, 468, 1953.

(IO7) C. S. Gum, Observatory, 72, I51, I952; 73, 123, 1953.

(I08) L. Biermann, Camb.

(109) L. E. Gurevich, in Problems of Cosmogony, 3, 94 (Moscow, 1954).

(I Io) H. Bondi, Mon. Not. R. Astr. Soc. 112, 195, 1952.

(I II) E. Schatzman, Camb.

(I I2) K. N. Dodd and W. H. McCrea, Mon. Not. R. Astr. Soc. 112, 205 and 374, 1952; I13, I62 and 484 , 1953 .

(I I3) G. Herbig et al., J.R. Astr. Soc. Can. 46, 222, 1952.

(I I4) K. G. Henize, $A p$. J. Ir9, 459, I954.

(II5) G. Haro et al., Bol. Obs. Tonantz. Tacubaya, nos. 7 and 8, 1953.

(II6) G. H. Herbig, $A p$. J. 113, 697, r95I.

(II7) G. Haro, $A p . J .115,572,1952$.

(1 I8) A. I. Lebedinsky, C.R. Acad. Sci. U.R.S.S. 84, no. 3, I952; 92, no. 3, I953.

(I I9) P. Swings, Ann. Astrophys. I5, 290, 1952.

(120) D. A. MacRae and J. Stock, Nature, Lond., r73, 589, 1954.

(I2I) L. H. Aller, R. Minkowski and O. C. Wilson, $A$ p. J. 120, 26I, I954.

(122) O. C. Wilson, $A p$. J. 117, 264, 1953.

(123) H. M. Johnson, $A p$. J. 120, 182, 1954.

(124) L. Rosino, Publ. Oss. Bologna, 6, no. 3, 1953.

(125) G. A. Shajn and V. M. Gaze, Publ. Crim. Obs. 11, 18, 1954.

(126) L. H. Aller, $A p$. J. I18, 547, 1953.

(127) M. J. Seaton, Mon. Not. R. Astr. Soc. I14, I54, 1954.

(128) M. J. Seaton, Proc. Roy. Soc. A, 218, 400, 1953.

(129) R. Minkowski, Publ. Astr. Soc. Pacif. 65, I6I, 1953.

(1 3о) A. van Pelt, Proc. Kon. Ned. Akad. Wet. 56, 67, 1953 (Amsterdam Circ. no. 7).

(13I) S. B. Pikelner, Publ. Crim. Obs. ro, r83, 1953.

(132) T. Hattori et al., Proc. Astr. Soc. Japan, 4, 152, 1953.

(133) K. Güssow, to appear in $Z$. $A p$.

(I34) H. Zanstra, B.A.N. no. 40I, I949.

(135) V. V. Sobolev, Astr. J. U.S.S.R. nos. 4 and 5, 1952.

(ז36) V. V. Sobolev, Bull. Leningrad Obs. (in the Press).

(137) W. Unno, Proc. Astr. Soc. Japan, 3, 158, 1952; 3, 178, 1952; 4, 100, 1952.

(138) W. Unno and K. Takakubo, Proc. Astr. Soc. Japan, 3, 195, 1952.

(139) B. Yada, Proc. Astr. Soc. Japan, 5, I28, 1953; 6, 67, 1954.

\title{
34a. SUB-COMMISSION FOR THE CATALOGUE OF DIFFUSE EMISSION NEBULAE
}

\section{Desirability of a new catalogue}

The use of cameras with a high aperture ratio and a wide field, combined with the possibility to isolate by means of very sensitive plates and red filters (sometimes multilayer interference filters) a narrow wave-length interval around $\mathrm{H} \alpha$ has enormously increased the possibilities for direct photography of emission nebulae. Many new emission nebulae have been discovered in the last few years. In addition, very faint emission was found to be present nearly everywhere at low galactic latitudes. The brighter nebulosities, among which are the emission nebulae in the usual sense as the brightest or most striking parts, are so crowded and interconnected in some regions of the sky that a division into 
discrete nebulae is, to some extent, arbitrary. All this makes it difficult at present to catalogue the emission nebulae. It seems nevertheless desirable to prepare a general catalogue, for

(a) Older catalogues and lists are very incomplete and often make no distinction between emission and reflection nebulae.

(b) As every investigator tends to use different criteria for the assignment of individual numbers, intercomparison of published lists is complicated.

(c) Much work is now being done on the connexion between emission nebulae and spiral structure and on investigations of the exciting stars for spectrum, magnitude and colour. In all this work the need for a common catalogue will be felt.

\section{Work of the sub-commission}

For these reasons Commission 34 adopted in 1952 a resolution 'to ask the Executive Committee to appoint a sub-committee in order to prepare a new catalogue of gaseous nebulae, preferably with reproductions and including the southern hemisphere'.

The members of the sub-commission have discussed this task by correspondence and in personal contacts. They have reached agreement on most points concerning the desirability and the technical possibilities of making a catalogue. They agreed that it was too early to propose definite plans for a new catalogue, but that a great deal could be learned from the current surveys and recently published lists of emission nebulae. The most important data are summarized below.

\section{Available surveys, lists and atlases}

The 48-inch Schmidt telescope at Mount Palomar provides at present the best and most complete material for a survey of diffuse nebulae in the northern sky. The prints of the red plates of the National Geographic Society-Palomar Observatory Sky Survey, that will be available in a few years, show quite faint nebulosity. Two partial studies of emission nebulae on Palomar Schmidt plates have already been made.

At the end of r953 S. Sharpless ( $r$ ) published a 'Catalogue of emission nebulae near the galactic plane', which is an important contribution: I42 discrete emission nebulae and six extended regions not divided into separate nebulae are given in the Catalogue. A comparison of the red and blue plates permitted to distinguish emission from reflection nebulae. The survey extends between galactic longitudes of $315^{\circ}$ and $105^{\circ}$, the limits in galactic latitude vary between $\mathrm{I}^{\circ}$ and $5^{\circ}$ on either side of the galactic equator. About half of the published nebulae had not been catalogued earlier. Owing to the large scale (I mm. $=67^{\prime \prime} \cdot \mathrm{r}$ ) Sharpless was able to discover a considerable number of small diffuse nebulae $\left(I-2^{\prime}\right)$. Besides the positions a short information concerning the diameter, form, structure and brightness of the nebulae are given in the Catalogue. The exciting stars associated with the nebulae are also noted.

A second plan was worked out by H. C. van de Hulst. In the beginning of 1954 he has investigated 2000 square degrees, roughly covering the area within $40^{\circ}$ to $140^{\circ}$ in longitude and $\pm 10^{\circ}$ in latitude. In this region 128 diffuse emission nebulae of smaller dimensions (from $0^{\prime} 4$ to $60^{\prime}$ in size) and twenty-eight extended objects larger than $I^{\circ}$ were listed. It is obvious that many of these objects were found earlier but no precise comparison with other references has yet been made. The prime aim of this investigation was to make a complete map with fairly accurate sketches of all emission details down to about $0^{\circ} \mathbf{I}$. No attempt at a more accurate determination of positions was made. It is planned to publish these maps together with a catalogue containing descriptive information on structure and on absorption and emission details.

This work also provided an estimate of the minimum time needed to make a complete catalogue covering all 879 fields of the Sky Survey. If the time needed for obtaining brightness estimates and accurate positions and for finding and cataloguing the exciting stars is not even taken into account, the complete work would take an experienced 
astronomer about two years. It is suggested that this work should be completed and published by steps.

Another project of interest for the sub-commission is being carried out at the Yerkes and McDonald Observatories by B. Strömgren, W. W. Morgan and H. L. Johnson. A survey is being made by means of a small Schmidt camera $(f=20 \mathrm{~cm}$.) with a field about $10^{\circ}$ in diameter. This instrument is especially suitable for faint extended objects. With a red filter of $270 \AA$ in width it has been used for a survey of the galactic belt between longitudes $303^{\circ}$ and $205^{\circ}$ and of many fields at higher galactic latitudes. As a first result a catalogue of 160 symmetrical nebulae has been prepared. We may expect further results when the narrow interference filters of $60 \AA$ width are used with the same camera. The faintest nebulae that have been observed have Emission Measures of about 300 .

Spectrographic observations of the galactic emission nebulae and regions by Johnson (2) with a nebular spectrograph furnish valuable information, particularly with regard to the emission measure.

An extensive $\mathrm{H} \alpha$ survey of the southern sky was carried out by the astronomers of Harvard College Observatory and Boyden Station (B. J. Bok, M. J. Bester and C. M. Wade). They used a 3 -inch $\mathrm{f} / \mathrm{I} \cdot 5$ Zeiss Sonnar in combination with a Baird narrow interference filter $(50 \AA)$ centred near $\mathrm{H} \alpha$ (transmission at $\mathrm{H} \alpha$ is about $70 \%$ ). The plate covers approximately 500 square degrees. In order to distinguish the emission nebulae and the extended $\mathrm{HII}$ regions from the reflection nebulae and from the stellar background, a second Baird narrow interference filter $(50 \AA)$ centred at $6480 \AA$ was used. Owing to the small scale $\left(\mathrm{rmm} .=30^{\prime}\right)$ the lower limit for recognizing the nebulae is about $4^{\prime}$. Two preliminary reports of this survey were issued in I953. A 'Catalogue of $\mathrm{HI}$ regions in the Milky Way for longitudes $250^{\circ}$ to $355^{\circ}$ (the galactic belt within $\pm 10^{\circ}$ in latitude) was completed (3). This catalogue contains $4 \mathrm{I}$ nebulae but often one number represents a complex of connected nebulosity. For instance, one number is given to the well-known nebulae NGC 65r4, NGC 6523 and some others. The co-ordinates for the faint extended HII regions are not given, but their boundaries are indicated by dotted lines in the accompanying charts. The majority of the nebulae, represented in the catalogue are new. A list of exciting stars and some other information are also given. The designation is based on the galactic co-ordinates and a classification in four types is given. The catalogue is accompanied by valuable identification charts and by eleven fine photographs, relating to nine large regions of the southern Milky Way. In addition, the astronomers of Harvard Observatory have given in their second interim report nineteen nebulae within $\mathrm{x} 74^{\circ}$ to $233^{\circ}$ galactic longitude.

Work on a joint Harvard and Armagh programme for the photography in blue and red light of the southern Milky Way between galactic longitudes $20^{\circ}$ and $330^{\circ}$ has begun at Boyden Station. The ADH 32 -inch telescope with $f=300 \mathrm{~cm}$. is used; the plate centres will be $4^{\circ}$ apart in longitude at galactic latitudes $-3^{\circ}, 0^{\circ}$, and $+3^{\circ}$.

A. D. Thackeray writes about the nebula work: 'Work on southern diffuse nebulae has been continued at the Radcliffe Observatory as opportunities occurred. In particular, spectra of the involved stars were investigated in order to reveal the sources of excitation. One remarkable case (reported also to Commission 29) is the detection of the heavily reddened Iom $^{\mathrm{m}} \mathrm{O}$-type star (HD type $\mathrm{G}_{5}$ ) within the diffuse nebula NGC 6334 (which is a radio source).'

In Australia, C. S. Gum and others (4) were surveying the southern Milky Way by means of a nebular spectrograph, and also by means of direct photography in $\mathrm{H} \alpha$ with a fast camera. During a stay in South Africa in I953-54 Whitford, Code and Houck photographed the southern sky with the aid of the Greenstein-Henyey camera, which was employed so effectively by Sharpless and Osterbrock (5) for studies of the northern Milky Way.

The study of nebulae started in 1949 at Simeis was continued. For the past three years a $640 \mathrm{~mm}$. camera $(\mathrm{f} / \mathrm{I} \cdot 4)$ has been used in combination with a red filter $\mathrm{KC}_{5}$. Other filters were also sometimes used to distinguish the emission nebulae. The field is nearly 
$3^{\circ}$ in diameter. Four lists of emission nebulae were published (6). The observers at Simeis have now listed zor objects, the majority of which are new. But it is necessary to note that here the designation is to some extent arbitrary. Separate numbers are often given to the conspicuous details of the same nebulosity. The same concerns the unknown details of a known nebula. A revised catalogue with somewhat reduced numbers will be published in the near future. Additional information, particularly concerning the exciting stars, will be included. Partly for that purpose observations are being carried out at the Simeis Observatory of the spectra of stars up to $12^{\mathrm{m} \cdot 2}$, by means of a $400 \mathrm{~mm}$. camera supplied with a $4 \mathrm{IO} \mathrm{mm}$. prism $\left(6^{\circ} 9\right)$. Also magnitudes, colour indices and colour excesses are being determined, mainly in the regions of emission nebulae. Simeis plates for the study of nebulae are mostly standardized and it is hoped to derive in future the values of the emission measure.

Extensive work on nebulae is being carried out at the Astrophysical Institute in Alma Ata (V. G. Fesenkov, D. A. Rozhkovski and others). The instrument used is the $500 \mathrm{mna}$. Maksutov menisc camera. The useful field is about $6^{\circ}$ in diameter. Different filters are being used, but mainly the red $\mathrm{KC}_{5}$ filters. For the most part the work is not in the domain of Sub-commission 34a, but a number of new emission nebulae were found and are to be included in the catalogue. D. Rozhkovski, V. Matiagin and G. Gavrilov are preparing a preliminary catalogue of luminous gaseous condensations.

An atlas of emission nebulae may be considered as a useful supplement to the catalogue of the nebulae. In this connexion it is necessary to mention the following three publications:

G. Shajn and V. Gaze, Atlas of diffuse gaseous nebulae (Moscow, 1952), containing 42 photographs.

V. G. Fesenkov and D. A. Rozhkovski, Atlas of gaseous-dust nebulae (Moscow, 1953), containing 40 photographs and 19 figures.

B. J. Bok, M. J. Bester and C. M. Wade, Atlas of the Southern Milky Way (Boston, 1954), containing II photographs.

G. ShaJN

President of the Sub-commission

\section{REFERENCES}

(I) S. Sharpless, $A p . J .118,362$, 1953.

(2) H. L. Johnson, $A p . J$. 118, 370, I953.

(3) B. J. Bok, M. J. Bester and C. M. Wade, Mem. Amer. Acad. Arts and Sci., 86, no. I, I955; Harvard Reprint $4 \mathrm{I} 6$.

(4) C. S. Gum et al., Observatory, 72, I 5 I, 1952; 73, I23, I953.

(5) S. Sharpless and D. E. Osterbrock, $A P . J .115,89$ and $140,1952$.

(6) First list: Publ. Crim. Astrophys. Obs. 6, 3, 1951; 35 nebulae. Second list: Publ. Crim. Astrophys. Obs. 7, 93, 1951; 87 nebulae. Third list: Publ. Crim. Astrophys. Obs. 9, 52, 1952; I 74 nebulae. Fourth list: Publ. Crim. Astrophys. Obs. 11, 39, 1954; 23 nebulae. Combined data of all lists: Publ. Crim. Astrophys. Obs. I5, I I, 1955. 
Report of the meeting. 3I August 1955

President: Prof. H. C. van de Hulst.

SeCRetary: Dr F. D. Kahn.

The President suggested that the meeting should discuss some points of particular interest in the Draft Report, since there was very little business to transact. The first such item was the distribution of interstellar matter within, say, 500 or $1000 \mathrm{ps}$. of the Sun.

Prof. Strömgren described preparations made at Yerkes and McDonald Observatories to use colour excesses of nearby $\mathrm{A}$ and $\mathrm{F}$ stars to plot the positions of interstellar dust clouds. Such stars are much more abundant than $\mathrm{O}$ and $\mathrm{B}$ stars and permit the discovery of much finer detail. The first need, though, is to measure accurately the colour indices of $\mathrm{A}$ and $\mathrm{F}$ stars. The photo-electric spectrograph now built at Yerkes can do this to within I\% for stars from type A2 to Go. With the 82-inch telescope an I8th magnitude star can be measured in three hours and a I3th magnitude star in Io minutes.

In answer to questions, Prof. Strömgren said that the dispersion used was $15 \AA / \mathrm{mm}$. and the bandwidth $40 \AA$. He thought the spectrograph was more flexible than an apparatus using interference filters, and the accuracy better than can be obtained with photographic plates. The sky background gives no trouble until the I8th magnitude.

The President then went on to discuss the work of Commission 34a. The need for a new catalogue of galactic nebulae was felt, but to make it would be difficult, for the distinction of individual nebulae is often arbitrary. The right time for making a new catalogue has not yet come. Dr Bok expressed the appreciation of the sub-committee members for the fine co-ordinating done by its chairman Dr Shajn. After a brief discussion of the relative merits of the new Palomar and Yerkes surveys of the sky the meeting decided unanimously: 'that Sub-committee 34a for the preparation of a new catalogue of galactic nebulae be discontinued, with thanks to its chairman and members for completing their report'.

Dr Courtès now spoke about the radial velocities of some emission nebulae.

The President then turned the discussion to the problem of interstellar temperatures. He described Spitzer's early work, his discovery of the great temperature difference between $\mathrm{H}$ I and $\mathrm{H}$ II regions and his prediction that this must have important dynamical effects.

Dr Spitzer said that his early estimates permitted a wide latitude of results. The usually quoted value $\left(50^{\circ} \mathrm{K}\right.$.) of the $\mathrm{H}$ I temperature might be too low (by about $75^{\circ} \mathrm{K}$.) because he had neglected some important sources of heating, e.g. mechanical heating and cosmic rays.

In answer to Prof. McCrea the President showed how the temperature of the $\mathrm{H}$ i gas is found from the maximum intensity of $2 \mathrm{r} \mathrm{cm}$. line measurements, and mentioned that, since the gas was probably unevenly heated, this must be the harmonic mean temperature.

Prof. Pikelner now spoke about his views on the gas between the clouds. With equipartition of energy, and a density of $0 \cdot 1$ atoms $/ \mathrm{cm}^{3}$ the atoms in this gas will have velocities of about $30 \mathrm{~km}$./s. and will therefore not remain close to the galactic plane. Some wide and shallow $\mathrm{Na}$ I and $\mathrm{Ca}$ II lines and the wings of the $2 \mathrm{I} \mathrm{cm}$. line give evidence for its existence. It will carry a magnetic field with it and cosmic ray particles moving in this will give some of the non-thermal radio background from our galaxy.

Prof. Spitzer then mentioned the probable existence of gas between clouds with $n \sim \mathrm{IO}^{-3}$ and $T \sim \mathrm{IO}^{6}{ }^{\circ} \mathrm{K}$. This gas would be unobservable. In connexion with Pikelner's idea we should test whether the wide $\mathrm{Ca}$ II and $\mathrm{Na}$ I lines are due to interstellar matter or stellar atmospheres, by using supergiant B stars belonging to spectroscopic binaries.

After a brief discussion on the origin of interstellar clouds, the meeting unanimously approved the Draft Report, and the President closed the meeting at 12 noon. 